\title{
Undergraduate and graduate programmes in astronomy for the developing world
}

\author{
John R. Percy \\ Department of Astronomy and Astrophysics \\ University of Toronto \\ Toronto ON Canada M5S 3H4 \\ email: jpercy@utm.utoronto.ca
}

\begin{abstract}
In this paper, I discuss some aspects of the design of undergraduate and graduate astronomy curricula, broadly defined, for developing countries. A fundamental requirement is to develop students' ability and desire to learn, both in university and beyond. I then discuss several aspects of the curriculum: (i) The programme of coursework in astronomy and related topics such as physics and mathematics; (ii) The associated practical and project work to develop skills as well as knowledge; (iii) Linking the coursework, effectively, to various aspects of research; (iv) Development of general academic and professional skills such as oral and written communication, teaching, planning and management, and the ability to function as part of an interdisciplinary team; and (v) Orientation to the culture of the university and to the science and the profession of astronomy.

To accomplish all of these goals may seem daunting, especially as many of them are not achieved in the most affluent universities. But much can be gained by recognizing that there are well-established "best practices" in education, achieved through formal education research, reflection, and experience. Simple resources, effectively used, can be superior to the highest technology, used without careful thought. It is often best to do a few things well; "less can be more". And effective partnership, both within the local university and with the outside astronomical community, can also contribute to success.
\end{abstract}

Keywords. Astronomy education, undergraduate and graduate curricula, developing countries

\section{Introduction}

Under "New Initiatives for Promoting Astronomical Education and Research in Developing Countries", the sub-title of the conference on which this book is based, the training of astronomers must rank highly. Very little of my presentation will be "new" to education professionals, but it may not be widely known, appreciated, or implemented by astronomers and by university professors, most of whom are amateurs in teaching and administration, in the sense that they have received little or no pre-service or in-service training in these fields.

In this paper, I reflect on the concept of best practices in designing undergraduate and beginning graduate programmes and courses. Many of the principles are very general, and would apply to any university, but I shall try to express them in terms of the needs of developing countries, as far as my limited experience is able.

There are many relevant papers on astronomy education in and for developing countries in two previous IAU conference proceedings, Pasachoff \& Percy (1990), and Gouguenheim et al. (1998), as well as in Batten (2001).

In truth, undergraduate programmes and courses at universities in the developed countries are far from perfect. The strategies for creating effective programmes and courses 
do not necessarily cost much money, so the developing countries have the opportunity to succeed - and exceed - by avoiding the errors that the rest of us have made.

I recognize the special challenges which face universities in the developing countries. These challenges are well described in this book, and in the proceedings of the previous conference on this subject (Batten 2001). The university (or the country!) may have only one astronomer, whose responsibilities and opportunities may be many, and who may have to teach several astronomy courses, over multiple terms, in multiple years. The astronomy programme will have to make use of other faculty members, in cognate departments, to teach other courses in the programme. Fortunately, many such faculty members have an interest in astronomy. But they must be willing to co-operate with each other. Universities tend to be compartmentalized into departments and groups which have only their own interests in mind. So negotiation, cooperation, and sharing are of utmost importance - and are usually beneficial for everyone. Education depends, more than anything else, on people, not equipment.

There is a general strategy for approaching this or any other education activity, which I like to express in terms of the Hodson diagram, since it was pictured very simply in a paper by the distinguished science educator Professor Derek Hodson (2001). We begin by defining the objectives of our astronomy programme and courses; we decide on a curriculum (which includes knowledge, skills, applications, and attitudes); we develop effective teaching strategies - which include much more than the lecture and the textbook. Most of all, we subject every part of this process to assessment, which includes both evaluation and feedback so that, every year, the programme and courses constantly improve.

The Hodson diagram emphasizes that we should first discuss the objectives of astronomy programmes and courses for the developing countries. Since there are so many aspects to astronomy, and so many applications of astronomy to public, school, and university education, to academic research, to the development of science and technology infrastructure, to communication with institutions and with the public, and to other practical matters, it follows that students in astronomy programmes should develop a broad range of knowledge, skills, and experiences. In developing countries, astronomers have to fulfil many roles.

The Hodson diagram also reminds us that educational activities such as the delivery of programmes and courses are subject to assessment - both evaluation and feedback just as our scientific observations and theories are. This can be done through formal education research, through a more simplified process called action research, or even more simply, through careful reflection. The problem is that very few university professors and administrators have any formal training in these or other aspects of education. Normally, they begin with what they experienced themselves, as undergraduates. They may reflect on ways to improve the experience - which is good. But teaching and learning in higher education is a well-developed discipline, with regular conferences and refereed journals. Sometimes the papers in these conferences and journals are rather esoteric, but there are individuals such as Tim Slater in the US who are working hard to apply this research to the reality of undergraduate programmes and courses. See Slater \& Adams (2003) for an excellent, inexpensive guide to teaching "Astro 101". And there is a free on-line journal Astronomy Education Review at http://aer.noao.edu.

The most important strategy for success is to develop students' ability and motivation to be self-learners - both in university and beyond. Often, students enter school or university with ability and motivation, but these are actually "turned off" by the system! One consequence, in the developed countries, is that both the strongest and the weakest students may fail in university. 
The student's success thus depends, not only on their innate intelligence, but on their attitude and other academic skills. It also depends on the attitude of the instructors and their university: on whether the education of students is of high priority. In this sense, the barriers for astronomy students in developing countries are high, but not necessarily insurmountable.

\section{The programme of coursework}

When one thinks of an undergraduate astronomy programme, one often thinks in terms of a list of courses. At one end of the spectrum, the list is long, and includes every possible sub-topic in astronomy, physics, mathematics - and possibly other disciplines such as statistics, and computer science as well. Most or all of the courses in the programme are specified.

At the other end of the spectrum, the student may take a much broader programme, especially in the first two years; this programme may include foundation courses in astronomy, physics, and mathematics, but it may also include many courses in the humanities and social sciences. This type of programme is favoured by many liberal-arts colleges and universities in the US.

Both approaches have some merit, and I tend to prefer the middle of the spectrum. At my university, 14 of 20 courses in the programme are specified. This leaves room for courses in useful cognate subjects, or courses that develop skills in management, teamwork, and communication. Some students use this course to complete a smaller programme in a second subject that interests them.

The students who enter the astronomy graduate programme at my university must have a strong background in physics. Usually they have had at least a course or two in astronomy, and/or some astronomy research experience. Occasionally, we have students who have come from a pure physics background. In that case, we want to be sure that they have a genuine interest and motivation for astronomy.

When astronomy courses are included in an undergraduate programme, they usually include one-term courses in: The Solar System, Sun and Stars, Galaxies and Cosmology and, if possible, Practical Astronomy. If the courses do not have a significant theoretical content, then there may be an additional course in Astrophysics. In addition, it is desirable to have an upper-year research project course. And there is often an upper-year "capstone" course in current or special topics. I have seen programmes with even more astronomy courses, but I would advise on fewer courses, rather than more. This kind of programme is probably good for students who will not be proceeding to graduate work, but will be proceeding directly to astronomy-related careers (such as teaching) which require understanding of a broad range of astronomy topics.

There is ongoing discussion in the education community about whether it is better to teach as much content as possible, or to teach less material but in more depth (and more effectively) - "less is more". To some extent, the actual curriculum of courses is less important than the general and specific skills and attitudes that the students learn.

\section{Practical and project work}

If you think back to my comment about curriculum, you will realize that courses tend to teach knowledge or content. The practical work and projects teach skills. They may also teach the applications of the content and skills to the real world, but this can be done even better through formal or informal internships. Students often intern with academics by carrying out a senior thesis or research project. If their programme has a 
more practical bent, they may serve an internship in industry, government, schools, or other real-world setting. This can be done as a course, or through summer employment. Internships can benefit the student, the university, and the supervisor or employer, but they must be carefully structured, supervised and evaluated by the university if they are to be effective.

There is another approach that is becoming very popular in education circles - community service learning (e.g. www. communityservicelearning.ca). This means carrying out a practical project, for course credit, under the joint supervision of a faculty member and a supervisor from the community. For example: the 1000 students in first-year Engineering at the University of Toronto undertake a practical design project in the community, usually in support of worthy non-profit community organizations.

How could undergraduate astronomy students, in developing countries, serve their communities? One way would be by assisting schoolteachers in the important task of teaching astronomy and physics. But there are many other possibilities. Elsewhere in this book, Patricia Rosenzweig describes a form of community service learning in which undergraduate students assist, enthusiastically, with school outreach activities.

Most science courses have an associated laboratory component. This can introduce students to equipment, data, software, and other analysis tools and techniques. But there is a real danger in what are called "cookbook labs" - ones in which students follow a specified procedure to obtain a specified result. This may explain why a study in the US showed that high school students who took physics labs (presumably of the cookbook variety) in school did more poorly in introductory university physics than students who had not taken such labs in high school. The expensive "black box" equipment, used in the developed countries, may actually be less effective than simpler, more transparent equipment.

\section{Linking coursework and research}

Linking coursework or teaching and research is a high priority in most North American universities. An influential document is the Boyer Report (1998) entitled Reinventing Undergraduate Education: A Blueprint for America's Research Universities. This concept includes giving students opportunities to develop research skills, and also exposing students to modern research as part of their coursework.

In my own university department, most of my colleagues are doing exciting frontier astronomy with large telescopes, satellites, or powerful computers. Very few of them feel that they are able or willing to supervise undergraduate research. So undergraduate research supervision falls to those of us who do relatively "small-science" projects such as my research on variable stars (Percy et al. 2006). Yet we are able to provide students with a genuine or authentic (to use the current education jargon) research experience, involving real data and analysis, resulting in a conference presentation or research paper with them as co-author. They may then go on and do a more high-powered senior thesis with one of the big-telescope astronomers.

An alternative is to give students projects which are not original, and are therefore not publishable, but which involve all the steps in a typical research project. These include:

- Development and understanding of the objectives and strategies for carrying out the project.

- Writing a project proposal, and periodic progress reports; this is a new experience for most students.

- Critical background reading of print and Internet sources, from the basic level to the research level. 
- Description of the data being used, including its origin, accuracy, and peculiarities.

- Description of the method(s) of analysis being used, including algorithms and software - their strengths, weaknesses, and peculiarities, and how they interact with the data.

- Testing of the analysis on trial data, to make sure that it produces a reasonable result.

- Analysis and interpretation of the new data, with appropriate output tables, graphs, and notes.

- Writing, editing, and defence of a final project report.

- Preparation of a poster paper, display, or oral presentation on the project.

The senior thesis, or major project, is a culmination of the development of the student's research knowledge and skills. While the project may be carried out one-on-one with a supervisor, it is important for the supervisor - or the course co-ordinator, if there are several students doing a senior project at the same time - to introduce some structured learning to the process. This structure can build upon the lower-level research skills, mentioned above.

At one time, it would have been difficult for a student in a developing country to do research at the senior undergraduate or graduate level, because of the lack of access to books, journals, and data. With the spread of the Internet, robotic telescopes, and data archives, this is gradually becoming less of a problem.

The lesson for the developing countries is thus that students can learn research by doing projects with small telescopes, or archival data that is available on-line. For instance, there are terabytes of variable star data available, with lots of science still to be "mined", along with freely-accessible software to analyze it. There are journals which do not charge an arm and a leg for page charges. Or the results can be self-published on-line.

\section{General academic skills}

Why, in this paper, am I putting so much emphasis on skills? One reason is that, a few years ago, a survey was done of $\mathrm{PhD}$ graduates of physical-science programmes in the US, 5 years after completing their education (Smith et al. 2002). They were asked to comment on how well their education prepared them for the job they were in. They indicated that they had been well-prepared for project design, analysis and interpretation of data, and for other aspects of problem-solving. But they had not been well prepared for: (i) oral and written communication; (ii) teaching, instructing, and mentoring; (iii) administering and managing projects and people; and (iv) working with individuals from different disciplines.

These are examples of general academic skills. Others are: autonomy, critical thinking, determination, enthusiasm, industriousness, initiative, teamwork, judgement, time management, and the ability to get al.ng with other people.

There is one highly-regarded method of instruction which can develop these skills very effectively, and that is problem-based learning - PBL (Albanese \& Mitchell 1993). Students work in groups to solve a significant practical problem, based on research, understanding, and agreement. PBL facilitates the formation of study groups, which have shown to be very effective in promoting learning. An undergraduate astronomy programme also leads to the formation of a cohort of students who take similar courses, share common experiences, and may well become lifelong colleagues, supporters, and friends. Large universities are just now discovering the benefits of organizing students into such cohorts or learning communities. 


\section{The student experience}

The current major concern of North American universities is something that goes beyond the formal curriculum - the student experience. Are the students satisfied with their experience? Surveys show that, especially in large universities, many students are uninterested and unengaged. Many are not motivated to learn and, even if they are, they face the barriers of large classes and untrained, uninterested instructors.

Here's where an astronomy programme in a developing country may have an advantage. It is unlikely that the astronomy, physics, and mathematics courses are large so - as long as the instructor is conscientious and genuinely interested in students - there is the potential for good learning, and good experience. How can this be encouraged? One way is by evaluating and rewarding the professors equally for their teaching, and research or professional work.

It's also important to evaluate the student experience by surveying students, anonymously if necessary. In my university, all courses are evaluated. The instructor may or may not act on the results. Programmes are not evaluated. If possible, graduates should be surveyed, both when they leave the university, and after they have been in the working world for a few years. And their comments should be taken seriously, and used to improve the programme and the experience.

\section{Graduate programmes and courses}

I will not comment on $\mathrm{PhD}$ programmes, since the structure is well-defined: the student carries out a significant original research project as an apprentice of the supervisor. Very often, the $\mathrm{PhD}$ is not done in the developing country; the student is sent overseas, to a more established institution, with more resources. However, the following general comments about MSc programmes have some relevance to all graduate work. And most of my comments about undergraduate programmes apply to graduate programmes as well.

In choosing students for graduate work, academic achievement is a necessary criterion, but not a sufficient one. My experience is that, as long as the student's average is B+ or greater (or the equivalent in your system), future success depends mostly on the student's general academic skills, attitudes, and motivation.

Whether entering an undergraduate programme or a graduate programme, students may have difficulty acclimatizing to the culture of the department and the university, especially if they come from another country. Formal and informal academic orientation and mentoring are absolutely essential - especially for students who come from a family or part of society without a tradition of university study - and are therefore highly recommended.

Although graduate students may have to take and pass courses - in which case, all of the above comments may apply - the essential feature of graduate education is supervised research. Just as university professors are amateurs at teaching, they are also amateurs at supervision. Students may have a supervising committee but, if the committee does not meet or take its work seriously, the student may fall by the wayside.

Graduate students should receive training in teaching, outreach, and communication, and should be encouraged to gain practical experience in these skills. These skills are essential for the students' future success. Practicing them can be a satisfying and motivating experience. And, because there are few astronomers in developing countries, it makes sense to use advanced students to contribute to teaching and outreach. 


\section{Becoming an astronomer - or not}

The topic of this book, and this paper, assumes that the students in the undergraduate astronomy program and courses will go on to become astronomers. Based on experience in both developing and developed countries, this may not be the case. There may be insufficient positions in astronomy for all the graduates. The students may develop skills which may lead them to non-astronomical jobs in government or industry. In some ways, it is useful to have astronomers in such positions of influence! We can't let government and industry be run entirely by lawyers who are scientifically illiterate! See Tobias et al. (1995) for an excellent discussion of science careers.

Unfortunately, universities in the developed countries often regard these students as failures, and do not maintain contact with them. This is unfortunate for many reasons. One is because they may be future donors to the university! They may also be useful mentors and role models for students who seek careers other than astronomy.

Astronomy is unique in that it can be continued as a hobby. So-called amateur astronomers can play important roles in astronomical teaching and research, and in providing grass-roots support for astronomy (Percy \& Wilson 2000). This may be especially important in developing countries. There is a tendency, in the developed countries, for astronomy departments to lose track of, and lose interest in graduates who leave astronomy. This is short-sighted - for many reasons.

\section{Epilogue}

It takes many years of patient but determined effort to build any new research or education structure, including an undergraduate or graduate astronomy programme; it does not happen overnight. IAU Commission 46 has a history of working with countries and institutions, on a long-term basis, to support the development of new astronomical centres. We look forward to working with you in the future.

\section{References}

Albanese, M.A. \& Mitchell, S. 1993, Academic Medicine, 68(1), 52

Batten, A.H. 2001, Astronomy for Developing Countries, (San Francisco: Astronomical Society of the Pacific)

Boyer Commission 1998, Reinventing Undergraduate Education: A Blueprint for America's Research Universities, at:

http://naples.cc.sunysb.edu/Pres/boyer.nsp/

Gouguenheim, L., McNally, D. \& Percy, J.R., 1998, New Trends in Astronomy Teaching, (Cambridge: Cambridge University Press)

Hodson, D. 2001, OISE Papers in STSE Education, Ontario Institute for Studies in Education, at the University of Toronto, 2,7

Pasachoff, J.M. \& Percy, J.R. 1990, The Teaching of Astronomy, (Cambridge: Cambridge University Press)

Percy, J.R. \& Wilson, J.B. 2000, Amateur-Professional Partnerships in Astronomy, (San Francisco: Astronomical Society of the Pacific Conference Series, 220).

Percy, J.R., Molak, A., Lund, H., Overbeek, D., Wehlau, A.F. \& Williams, P.F. 2006, Publ. Astron. Soc. Pacific, 118, 805

Slater, T.F. \& Adams, J.P. 2003, Learner-Centered Astronomy Teaching: Strategies for Astro 101, (Upper Saddle River NJ: Pearson Education Ltd.)

Smith, S.J. et al. 2002, Am. J. Phys., 70 (11), 1081

Tobias, S., Aylesworth, K. \& Chubin, D.F. 1995, Rethinking Science as a Career (Tucson AZ: The Research Corporation) 


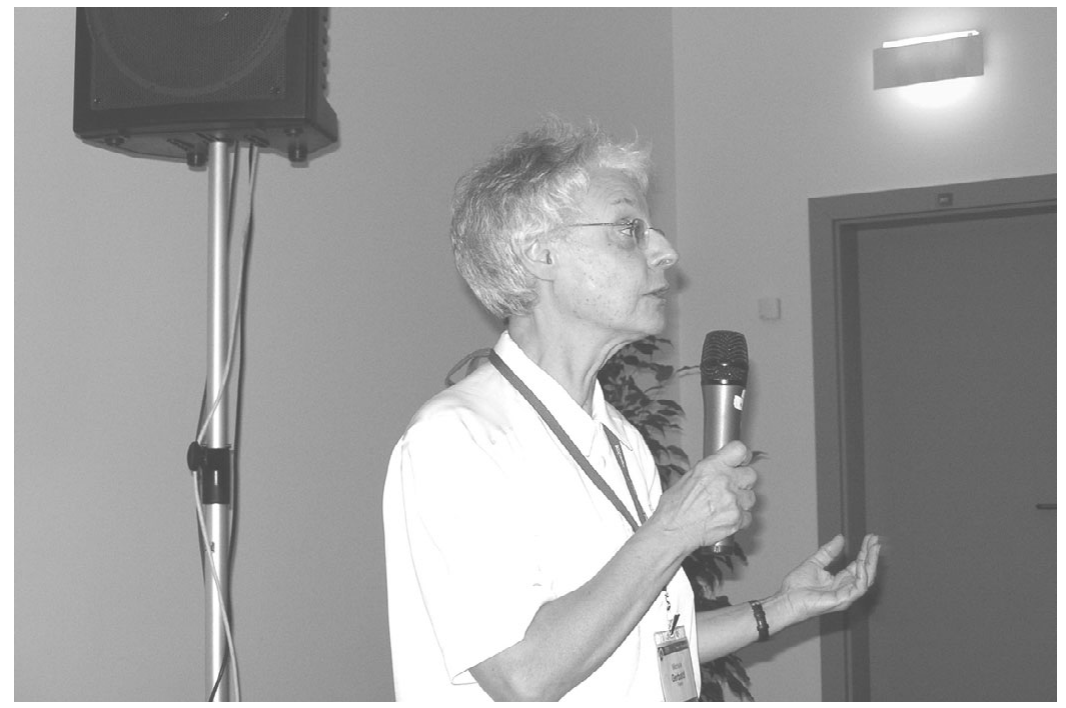

Michèle Gerbaldi

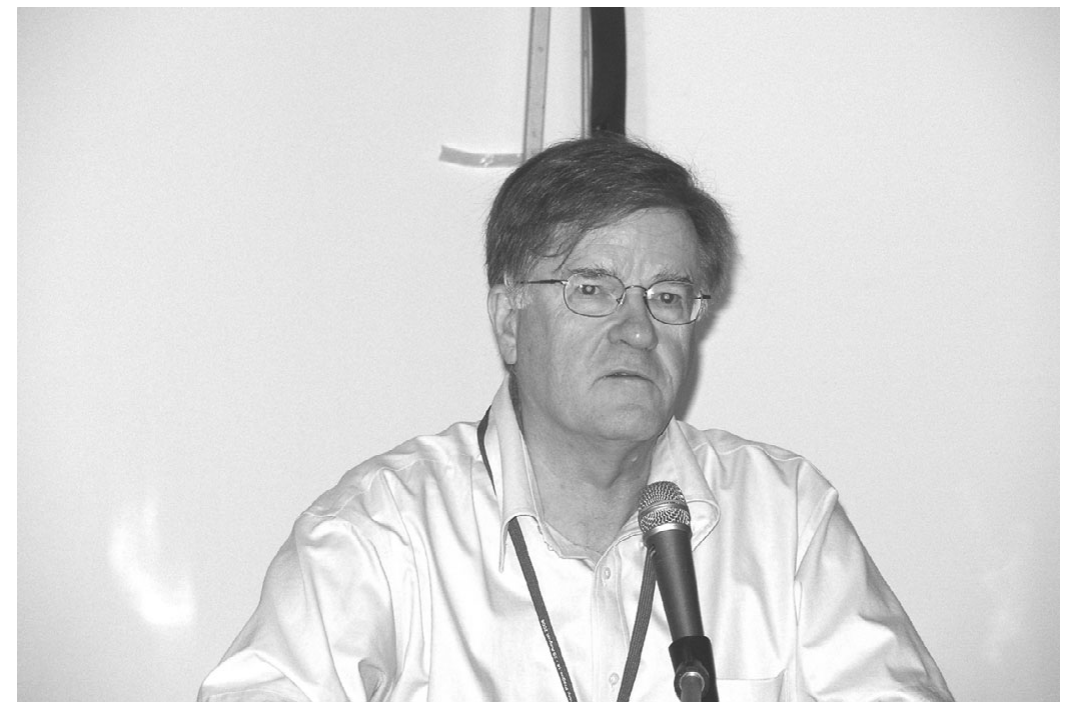

John Percy 